\title{
Effects of relay position and power allocation in space-time coded cooperative wireless systems
}

\author{
Luca Zuari, Andrea Conti, and Velio Tralli \\ ENDIF - University of Ferrara - CNIT \\ via Saragat, 1 - 44100 Ferrara - Italy \\ lzuari@libero.it, a.conti@ieee.org, vtralli@ieee.org
}

\begin{abstract}
Cooperative communications are effective in improving the performance and extend the coverage of wireless networks. One issue is to find proper methods to allocate cooperative nodes. In this paper we investigate the effects of relay position and power allocation strategy in cooperative communications employing space-time codes (STCs). We consider non-ideal links between source, relay, and destination enabling the analysis of relay allocation problem based on the performance of each link in realistic scenarios. The frame error rate for various channel conditions, available diversity, relays position, and transmitted power levels is obtained. Both the situation of balanced and unbalanced transmit power levels for source, relay, and destination are compared. Cooperative pragmatic STC in block fading channel (BFC) are considered for our analysis. The results provide insights on how to allocate relay nodes based on geometry, link quality, and transmitted power considerations.
\end{abstract}

\section{INTRODUCTION}

Cooperative communications are gaining increasing interest as a new communication paradigm involving both transmission and distributed processing which promises significant increase of capacity and diversity gain in wireless networks, by counteracting fading channels with cooperative diversity.

Several issues arise with cooperative diversity schemes such as, among others, channel modeling and implementation aspects [1], [2], protocols and resource management [3], the choice of proper relays [4], power allocation among cooperating nodes [5], and cooperative/distributed STCs [6], [7].

In addition to physical antenna arrays, the relay channel model enables the exploitation of distributed antennas belonging to multiple relaying terminals. This form of space diversity is referred to as cooperative diversity because terminals share antennas and other resources to create a virtual array through distributed transmission and signal processing [8], [9].

With the introduction of STCs it has been shown how, with the use of proper trellis codes, multiple transmitting antennas can be exploited to improve system performance obtaining both diversity and coding gain, without sacrificing spectral efficiency [10]. In [11] a pragmatic approach to STCs, called pragmatic space-time codes (P-STCs), has been proposed: it simplifies the encoder and decoder structures and also allows a feasible method to search for good codes in BFC (PSTC consists in the use of standard convolutional encoders and Viterbi decoders over multiple transmitting and receiving antennas).

In [12] a design methodology of P-STCs for relay networks was provided, resulting in increased flexibility with respect to the above issues. The channel between each transmitting and receiving antennas pair is modeled as BFC which includes a variety of fading rates, from fast fading (i.e., ideal symbol interleaving) to quasi-static [13].

Typically, the link between relay and destination is assumed as an independent, identically distributed (i.i.d.) version of the link between source and destination just adding diversity advantage. In our framework presented here we look carefully on the quality of the links involving the relay (both source-torelay and relay-to-destination) with two-folds goal: (i) evaluate the performance in a more realistic scenario where also the relay's position impacts the effectiveness of cooperation and the performance at the destination, and (ii) provide some insight on how to choose relay nodes based on both geometrical and link quality conditions, and power utilization. The latter point is relevant when power consumption at each node or interference issues have to be addressed.

\section{System Model}

The cooperative scheme is depicted in Fig. 1 and follows time-division channel allocations with orthogonal cooperative diversity transmission [6]. The source $S$ divides the time-slot in two equal segments, the first from time $t_{1}$ to $t_{1}+\Theta$ and the second from $t_{2}=t_{1}+\Theta$ to $t_{2}+\Theta$, where $\Theta$ is the segment duration. In the first segment the source broadcasts its coded symbols, in the second segment all the active relays (which are able to decode the message) forward the information through proper encoding to take advantage of the total available diversity. Thus, the design of proper STCs for the two phases is crucial to maximize both achievable diversity and coding gain.

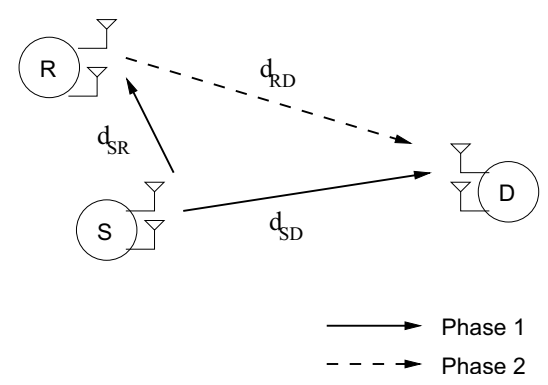

Fig. 1. Two-phase relaying scheme: phase 1 (continuous line), phase 2 (dashed line). Source, relays and destination nodes are denoted with S, R, D, respectively. 
We assume $n$ transmitting antennas at each terminal, $m_{r}$ and $m_{\mathrm{D}}$ receiving antennas at the relay $r$ and at the destination, respectively. Hence, $n_{1}=n$ transmitting antennas will be used in the first phase and a total of $n_{2}=R n$ transmitting antennas will be used in the second phase, where $R$ is the number of potential relays. The relay is initialized at the beginning of a data communication session and is kept unchanged over the session. We also assume, to simplify signaling, that the source does not know whether the transmission to relays is successfull or not, hence it does not transmit in the second time slot.

We indicate with $c_{r, i}^{(t)}$ the modulation symbol transmitted by relay $r$ ( $0 \leq r \leq R$, and $r=0$ is the source) on the antenna $i$ at discrete time $t$ (i.e. at the $t^{\text {th }}$ instant of the encoder clock). Each symbol is assumed to have unit norm and to be generated according to the modulation format by suitable mapping. Note that symbol $c_{0, i}^{(t)}$ is transmitted at time $t_{1}+t$, while symbols $c_{r, i}^{(t)}$ for $r>0$ are transmitted at time $t_{2}+t$. In the first phase, symbols $c_{0, i}^{(t)}$ are received by each relay; if correctly decoded ${ }^{1}$ then the relay re-encodes and forwards to the destination. The received signals corresponding to all symbols $c_{r, i}^{(t)}$ are jointly decoded by the destination at the reference time $t$. We also denote with $\mathbf{C}^{(t)}$ a super-symbol, which is the vector of the $(R+1) n$ outputs of the "virtual encoder" constituted by source and relays encoders. A codeword is a sequence $\underline{c}=\left(\mathbf{C}^{(1)}, \ldots, \mathbf{C}^{(N)}\right)$ of $N$ super-symbols generated by the source and relays' encoders. This codeword $\underline{c}$ is interleaved before transmission to obtain the sequence $\underline{c}_{I}=\mathcal{I}(\underline{c})=$ $\left(\mathbf{C}^{\left(\sigma_{1}\right)}, \ldots, \mathbf{C}^{\left(\sigma_{N}\right)}\right)$, where $\sigma_{1}, \ldots, \sigma_{N}$ is a permutation of the integers $1, \ldots, N$ and $\mathcal{I}(\cdot)$ is the interleaving function. Note that with this notation the permutation is the same for all the transmitting terminals in the two phases.

The channel model includes additive white Gaussian noise (AWGN) and multiplicative flat fading, with Rayleigh distributed amplitudes assumed constant over blocks of $B$ consecutive transmitted space-time symbols and independent from block to block [13]. Perfect channel state information is assumed at the decoder for each node ${ }^{2}$, whereas the transmitters only have to know mean channel gain for power allocation.

For the destination D the transmitted super-symbol at time $\sigma_{t}$ goes through a compound channel described by the $\left(n_{1}+\right.$ $\left.n_{2}\right) \times m_{\mathrm{D}}$ channel matrix $\mathbf{H}^{\left(\sigma_{t}, \mathrm{D}\right)}=\left[H_{0}^{\left(\sigma_{t}, \mathrm{D}\right)}, \ldots, H_{R}^{\left(\sigma_{t}, \mathrm{D}\right)}\right]^{T}$ where $H_{r}^{\left(\sigma_{t}, \mathrm{D}\right)}=\left\{h_{r, i, s}^{\left(\sigma_{t}, \mathrm{D}\right)}\right\}$, and $h_{r, i, s}^{\left(\sigma_{t}, \mathrm{D}\right)}$ is the channel gain between transmitting antenna $i(i=1, \ldots, n)$ of the terminal $r$ and receiving antenna $s$ at the destination $\mathrm{D}\left(s=1, \ldots, m_{\mathrm{D}}\right.$ at time $\sigma_{t}$ ).

In the BFC model these channel matrices do not change for $B$ consecutive transmissions, hence we actually have only $L=N / B$ possible distinct channel matrix instances per codeword $^{3}$. Denoting by $\mathcal{Z}=\left\{\mathbf{Z}_{1}, \ldots, \mathbf{Z}_{L}\right\}$ the set of $L$ channel instances, we have $\mathbf{H}^{\left(\sigma_{t}, \mathrm{D}\right)}=\mathbf{Z}_{l}$ for $\sigma_{t}=(l-1) B+1, \ldots, l B$ and $l=1, \ldots, L$. When the fading block length, $B$, is equal

\footnotetext{
${ }^{1}$ We assume CRC perfectly recognizing if a codeword is correctly decoded. ${ }^{2}$ As well known, nonperfect CSI at the receiver leads to some performance degradation, but this is not whithin the scope of paper investigation.

${ }^{3}$ For the sake of simplicity we assume $N$ and $B$ such that $L$ is an integer.
}

to one, we have the ideally interleaved fading channel (i.e., independent fading levels from symbol to symbol), while for $L=1$ we have the quasi-static fading channel (fading level constant over a codeword); by varying $L$ we can describe channels with different correlation degrees [13].

Similarly, in the first phase, the $r \underline{\text { th }}$ relay $\mathrm{R}_{r}$ experiences a channel described by the $\left(n_{1} \times m_{r}\right)$ channel matrix $H_{0}^{\left(\sigma_{t}, \mathrm{R}_{r}\right)}=$ $\left\{h_{i, s}^{\left(\sigma_{t}, \mathrm{R}_{r}\right)}\right\}$ where $h_{i, s}^{\left(\sigma_{t}, \mathrm{R}_{r}\right)}$ is the channel gain between transmitting antenna $i(i=1, \ldots, n)$ of the source and receiving antenna $s$ at the relay $\mathrm{R}_{r}\left(s=1, \ldots, m_{r}\right)$ at time $\sigma_{t}$.

At the destination the sequence of received signal vectors is $\left(\mathbf{R}^{\left(\sigma_{1}, \mathrm{D}\right)}, \ldots, \mathbf{R}^{\left(\sigma_{N}, \mathrm{D}\right)}\right)$, and after de-interleaving we have $\left(\mathbf{R}^{(1, \mathrm{D})}, \ldots, \mathbf{R}^{(N, \mathrm{D})}\right)$, where the received vector at time $t$ is $\mathbf{R}^{(t, \mathrm{D})}=\left[r_{1}^{(t, \mathrm{D}, 1)} r_{1}^{(t, \mathrm{D}, 2)} \cdots r_{m_{\mathrm{D}}}^{(t, \mathrm{D}, 1)} r_{m_{\mathrm{D}}}^{(t, \mathrm{D}, 2)}\right]^{T}$ with
$r_{s}^{(t, \mathrm{D}, 1)}=\sqrt{E_{0}} \sum_{i=1}^{n} h_{0, i, s}^{(t, \mathrm{D})} c_{0, i}^{(t)}+\eta_{s}^{(t, \mathrm{D}, 1)}$

in the first phase $\left(s=1, \ldots, m_{\mathrm{D}}\right)$ and

$$
r_{s}^{(t, \mathrm{D}, 2)}=\sum_{r=1}^{R} \sqrt{E_{r}} \sum_{i=1}^{n} h_{r, i, s}^{(t, \mathrm{D})} c_{r, i}^{(t)}+\eta_{s}^{(t, \mathrm{D}, 2)}
$$

for the second phase. In this equation $r_{s}^{(t, \mathrm{D}, l)}$ is the signalspace representation of the signal received by antenna $s$ at time $t$ in phase $l$, the noise terms $\eta_{s}^{(t, \mathrm{D}, l)}$ are i.i.d. complex Gaussian random variables (r.v.s), with zero mean and variance $N_{0} / 2$ per dimension, and the r.v.s $h_{r, i, s}^{(t, D)}$ represent the deinterleaved complex Gaussian fading coefficients. Similarly, the received signal vector at the $r$ th relay in phase 1 at time $t$ is $\mathbf{R}^{\left(t, \mathrm{R}_{r}\right)}=\left[r_{1}^{\left(t, \mathrm{R}_{r}\right)} \cdots r_{m_{r}}^{\left(t, \mathrm{R}_{r}\right)}\right]^{T}$ with components

$r_{s}^{\left(t, \mathrm{R}_{r}\right)}=\sqrt{E_{0}} \sum_{i=1}^{n} h_{i, s}^{\left(t, \mathrm{R}_{r}\right)} c_{0, i}^{(t)}+\eta_{s}^{\left(t, \mathrm{R}_{r}, 1\right)}, \quad s=1, \ldots, m_{\mathrm{R}_{r}}$.

We assume spatially uncorrelated channels with elements $h_{r, i, s}^{(t, \mathrm{D})}$ and $h_{i, s}^{\left(t, \mathrm{R}_{r}\right)}$ independent, non-identically distributed (i.n.i.d.) Complex Gaussian r.v.s with zero mean and variance per dimension given by

$$
\begin{cases}1 / 2 & \text { for } h_{0, i, s}^{(t, \mathrm{D})} \\ \Delta_{\mathrm{R}_{r} \mathrm{D}} / 2 & \text { for } h_{r, i, s}^{(t, \mathrm{D})} \\ \Delta_{\mathrm{SR}_{r}} / 2 & \text { for } h_{i, s}^{\left(t, \mathrm{R}_{r}\right)}\end{cases}
$$

where

$$
\begin{aligned}
\Delta_{\mathrm{SR}_{r}} & =\left(d_{\mathrm{SR}_{r}} / d_{\mathrm{SD}}\right)^{-\beta} \\
\Delta_{\mathrm{R}_{r} \mathrm{D}} & =\left(d_{\mathrm{R}_{r} \mathrm{D}} / d_{\mathrm{SD}}\right)^{-\beta} .
\end{aligned}
$$

Here, $d_{\mathrm{SD}}$ is the distance between source and destination, $d_{\mathrm{SR}_{r}}$ is the distance between source and relay $\mathrm{R}_{r}$, and $d_{\mathrm{R}_{r} \mathrm{D}}$ is the distance between relay $\mathrm{R}_{r}$ and destination; at the distance $d$ a path-loss proportional to $d^{\beta}$ is assumed.

The average transmitted energy per symbol $E_{\mathrm{s}}$, when all relays are active, is equal to

$$
E_{\mathrm{s}}=\sum_{r=0}^{R} E_{r} /(R+1)
$$


The energy transmitted per information bit is $E_{\mathrm{b}}=E_{\mathrm{s}} /\left(h R_{\mathrm{c}}\right)$ where $h$ is the number of bits per modulation symbol and $R_{\mathrm{c}}$ is the code-rate of the cooperative space-time code.

As far as power allocation among source and relays is concerned (i.e., the values of $E_{r}$ ) we consider two different strategies:

- Uniform power allocation: the source and all relays transmit with equal power thus $E_{r}=E_{\mathrm{s}}$ for $r=0,1, \ldots, R$;

- Ideal power control: the power among source and relays are balanced such that the average received power at the destination is the same. Thus, the source transmits with $E_{0}$ and the $r$-th relay with $E_{r}=E_{0} / \Delta_{\mathrm{R}_{r} \mathrm{D}}$ where

$$
E_{0}=E_{\mathrm{s}}(R+1) /\left(1+\sum_{r=1}^{R} 1 / \Delta_{\mathrm{R}_{r} \mathrm{D}}\right) .
$$

\section{COOPERATIVE SPACE-TIME CODE FOR RELAYING}

In the case of the two-phase relaying scheme shown in Fig.1, the probability of transmission failure over the two phases depends on the number of relays available for cooperation and on the quality of links source-destination, sourcerelays, and relays-destination.

Depending on terminals' positions the relays are set by looking at those that are able to guarantee effective cooperation with the source and to satisfy the target performance at the destination. Sometimes, due to fast fading fluctuations, it may happen that a relay is not able to decode the source codewords in the first phase. Let us denote by $P_{\mathrm{e}}^{(\mathrm{SD})}$ the error probability for source-destination link, $P_{\mathrm{e}}^{\left(\mathrm{SR}_{r}\right)}$ the error probability for the source- $r \underline{\text { th }}$ relay link, and with $P_{\mathrm{e}}^{(\mathrm{S} \mathcal{R} \mathrm{D})}$ the error probability for the link from the source plus active relays $(\mathcal{R}$ being the set of active relays) to destination. Note that these performance metrics are functions of system parameters as

$$
\begin{aligned}
P_{\mathrm{e}}^{(\mathrm{SD})} & =f^{(\mathrm{SD})}\left(E_{\mathrm{s}}, N_{0}\right), \\
P_{\mathrm{e}}^{\left(\mathrm{SR}_{r}\right)} & =f^{\left(\mathrm{SR}_{r}\right)}\left(E_{\mathrm{s}}, \Delta_{\mathrm{SR}_{r}}, N_{0}\right), \\
P_{\mathrm{e}}^{(\mathrm{S} \mathcal{R} \mathrm{D})} & =f^{(\mathrm{S} \mathcal{R} \mathrm{D})}\left(E_{\mathrm{s}},\left\{\Delta_{\mathrm{R}_{r} \mathrm{D}}\right\}, N_{0}\right) .
\end{aligned}
$$

The error probability at destination for one relay is given by

$$
P_{e}=P_{e}^{\left(\mathrm{SR}_{1}\right)} P_{e}^{(\mathrm{SD})}+\left(1-P_{e}^{\left(\mathrm{SR}_{1}\right)}\right) P_{e}^{\left(\mathrm{SR}_{1} \mathrm{D}\right)} .
$$

This can be generalized for multiple relays. As an example, for two relays it results

$$
\begin{aligned}
P_{e} & =P_{e}^{\left(\mathrm{SR}_{1}\right)} P_{e}^{\left(\mathrm{SR}_{2}\right)} P_{e}^{(\mathrm{SD})}+\left(1-P_{e}^{\left(\mathrm{SR}_{1}\right)}\right) P_{e}^{\left(\mathrm{SR}_{2}\right)} P_{e}^{\left(\mathrm{SR}_{1} \mathrm{D}\right)} \\
& +P_{e}^{\left(\mathrm{SR}_{1}\right)}\left(1-P_{e}^{\left(\mathrm{SR}_{2}\right)}\right) P_{e}^{\left(\mathrm{SR}_{2} \mathrm{D}\right)} \\
& +\left(1-P_{e}^{\left(\mathrm{SR}_{1}\right)}\right)\left(1-P_{e}^{\left(\mathrm{SR}_{2}\right)}\right) P_{e}^{\left(\mathrm{SR}_{1} \mathrm{R}_{2} \mathrm{D}\right)}
\end{aligned}
$$

For the goal of our paper, we consider space-time trellis codes for relaying networks by using the pragmatic approach of [11]. The pragmatic approach uses a low-complexity architecture for STC where the code components are built by the concatenation of a binary convolutional encoder and binary phase shift keying (BPSK) or quaternary phase shift keying (QPSK) modulator. The $k$ information bits are encoded by a convolutional encoder with rate $k /(n h)$ and the $n h$ output bits are divided into $n$ streams, one for each transmitting antenna, of BPSK $(h=1)$ or QPSK $(h=2)$ symbols that are obtained from a natural (Gray) mapping of $h$ bits. Then, each stream of symbols is eventually interleaved. If $\mu$ is the encoder constraint length then the associated trellis has $2^{k(\mu-1)}$ states.

We can describe a P-STC for cooperative communication, obtained by joining the $R+1$ code components used by the cooperating transmitters, by using the trellis of each encoder (the same as for the convolutional codes), labelling the generic branch from state $S_{i}$ to state $S_{j}$ with the super-symbol $\widetilde{\mathbf{C}}_{S_{i} \rightarrow S_{j}}=\left[\widetilde{c}_{0,1}, \ldots, \widetilde{c}_{R, n}\right]^{T}$, where for BPSK, the symbol $\widetilde{c}_{r, i}$ is the output (in antipodal form) of the $i \underline{\text { th }}$ generator of the $r$ th transmitter. One of the advantages of the pragmatic architecture is that the maximum likelihood (ML) decoder is the usual Viterbi decoder for the convolutional encoder adopted (same trellis), with a simple modification of the branch metrics. Being $\left\{\widetilde{c}_{r, i}\right\}$ the set ofoutput symbols labelling the branch, the branch metric for the Viterbi decoder is

$$
\begin{aligned}
& \sum_{s=1}^{m_{\mathrm{D}}}\left(\left|r_{s}^{(t, \mathrm{D}, 1)}-\sqrt{E_{0}} \sum_{i=1}^{n} h_{0, i, s}^{(t, \mathrm{D})} \widetilde{c}_{0, i}\right|^{2}\right. \\
& \left.+\left|r_{s}^{(t, \mathrm{D}, 2)}-\sum_{r=1}^{R} \sqrt{E_{r}} \sum_{i=1}^{n} h_{r, i, s}^{(t, \mathrm{D})} \widetilde{c}_{r, i}\right|^{2}\right) .
\end{aligned}
$$

In [12] it was discussed how to perform an efficient search for generators of cooperative P-STCs in BFC. For the design of the coding scheme with cooperative relays it is generally recognized that the code components used by the source in phase 1 should maximize diversity and coding gain for each link connecting the source to relays and destination. The other code components should be designed to maximize diversity and coding gain of the entire cooperative code, that is the code including all the code components transmitted during phase 1 and 2, for any possible number of cooperative relays [14].

By assuming that the cooperative code is obtained by joining code components in phase 2 from every relay able to decode the source message, the code may be designed as STCs with overlay construction. For P-STCs this gives cooperative overlay pragmatic space-time codes (COP-STCs). With this method, a good code for $R$ relays is designed starting from a good code for $R-1$ relays and by adding the best code component that maximize diversity and coding gain of the final code. In this way the first code component used by the source in phase 1 is always a good code. In the case of a fixed set of more than one cooperating relays the sequence of predesigned additional code components can be assigned to the relays ranked in order of average link quality, so that they are used with high probability in the same order for which they have been designed.

\section{Numerical Results and Relay Allocation}

The performance figure we are interested in is the mean frame error rate (FER) at the destination averaged over rapid processes, such that those related to BFC, and the effective 


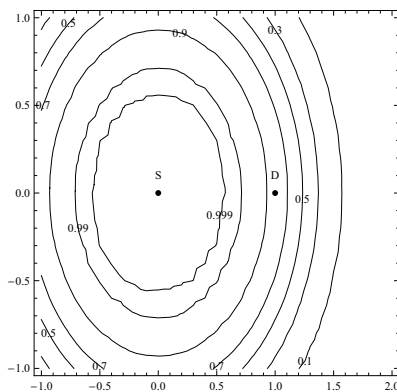

(a) $P_{\text {coop }}$ contours for $L=1$ with uniform power allocation.

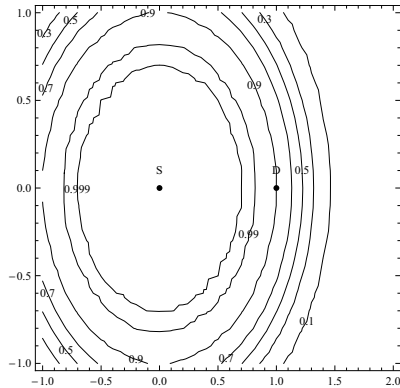

(b) $P_{\text {coop }}$ contours for $L=5$ with uniform power allocation.

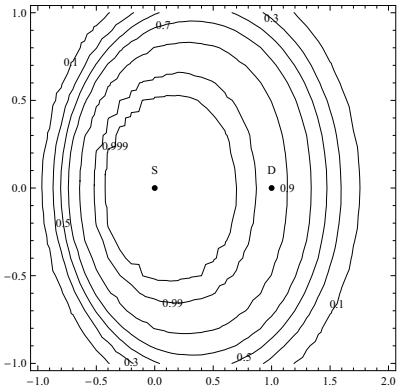

(c) $P_{\text {coop }}$ contours for $L=1$ with ideal power control.

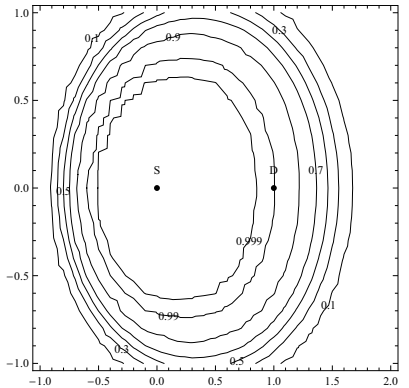

(d) $P_{\text {coop }}$ contours for $L=5$ with ideal power control.

Fig. 2. Spatial distribution of $P_{\text {coop }}$ depending on relay's position. BFC for $E_{\mathrm{b}} / N_{0}=5 \mathrm{~dB}$.

relay cooperation. We evaluate it as a function of signal-tonoise ratio (SNR), relay location, $\mathrm{BFC}$ characteristics, and power allocation strategy, for COP-STC. Then we discuss the implication on relay allocation. We refer to a scenario in which a source transmits to a destination with one potential relay (e.g., this is the situation for the downlink of cellular systems where the source is the base-station, the destination is the mobile-station, and the relay is a terminal enabled to act as a cooperating node placed in any position over the space).

The results are obtained for BPSK constellation signaling, COP-STC with 8 states, code-rate $1 / 4$, generators $(13,15,11,17)_{8}$ as from [12], $N=130, n=2$ transmitting antennas per node, $m_{\mathrm{R}}=2$ receiving antennas at the relay, $m_{\mathrm{D}}=1$ receiving antenna at the destination, and with the two power allocation strategies discussed in Sec. II. Various BFCs (i.e., values of $L$ ) are considered. The SNR is defined as $E_{\mathrm{b}} / N_{0}$ per receiving antenna element where, for a fair comparison among situations with different number of relays, $E_{\mathrm{b}}$ is the total energy per information bit over all transmitting nodes and averaged with respect to fading. All possible relay's positions on a bi-dimensional scaled plane (source in coordinates $(0,0)$ and destination in $(1,0))$, are evaluated with distances normalized to $d_{\mathrm{SD}}$ and path-loss coefficient $\beta=3.5$ which is feasible choice for many wireless scenarios.

\section{A. Spatial Distribution of the Cooperation Probability}

Fig. 2 shows the probability of relay's cooperation $P_{\text {coop }}$ for various relay's positions with the two power allocation strategies. It is defined as $P_{\text {coop }}=1-P_{e}^{(\mathrm{SR})}$. As expected the cooperation probability is large when the relay is placed around the source and as $L$ increases. When ideal power control is adopted the regions with high probability of cooperation move toward the destination. Of course, the spatial distribution of the cooperation probability is only one aspect; in fact the cooperation aims at minimizing the FER at the destination. Situations in which the relay always cooperates but it has a very bad link quality with the destination or, viceversa, it would really improve the performance but due to its link with the source rarely cooperates, have to be avoided.

\section{B. Frame Error Rate at the Destination}

The FER at the destination is obtained by simulating single terms of (7) depending on relay's position, SNR, BFC, and power allocation. In Fig. 3 the FER with uniform power allocation is shown for $L=1,5,130$, and $E_{\mathrm{b}} / N_{0}=5 \mathrm{~dB}$. In red dash the FER for the link source-to-destination in the absence of relaying is shown to understand where the cooperative relay improves the performance. We can note that the region where cooperation is beneficial is large and centered in a point close to the destination. Contours enlarge, as well as the minimum FER decreases, when $L$ increases. Results for $L=5$ and $L=130$ are overlapped since the number of states of the chosen COP-STC saturates the available timediversity. The results enable the comparison of the FER in the presence of cooperation with the one without cooperation, and also provide a geometrical view of where the best relay should be located. The same kind of results but for ideal power control can be seen in Fig. 4. Here, the main difference due to the power allocation strategy is given by the fact that the minimum FER is greater with ideal power control but at the same time the FER is less sensitive to the relay's position.

Finally, it should be underlined that in the case of ideal power control the transmitted power decreases as the relay is close to the destination. For the example application discussed above, when the destination is near to the cell edge, this causes a lower level of intercell interference from the relay. In general, these kind of results enable to understanding power consumption of relay and represent an input for the analysis of the interference caused by relay.

\section{Relay Allocation Strategy}

The presented results provide insights useful to define methods for relay assignment. The choice of relay's position depends on a proper balancing between the FER at the destination and the transmitted power at the relay. We summarize the following considerations: (i) the relay should be located between source and destination, preferably closer to the destination than to the source; (ii) by using ideal power control the benefits of cooperation on the FER are less sensitive to the relay's position; (iii) with ideal power control the power level transmitted by the relay is lower near the destination. Thus, even if the probability of cooperation increases as the relay is close to the source, the performance metrics indicate that the goodness of the link relay-destination is important and the cooperation beneficial regions are closer 


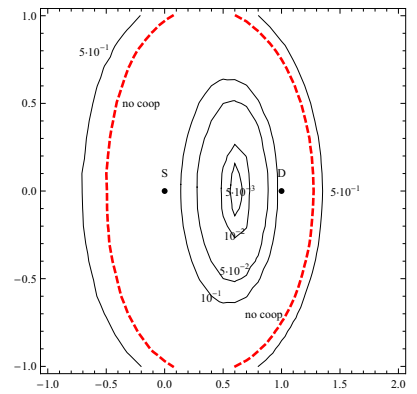

(a) FER contours for $L=1$.

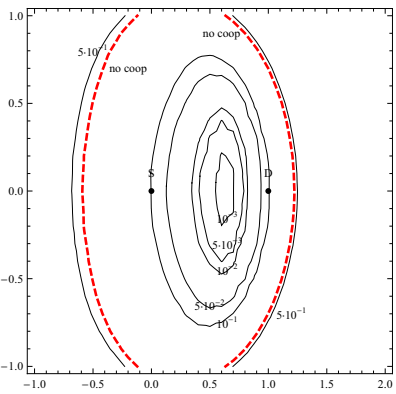

(b) FER contours for $L=5$.

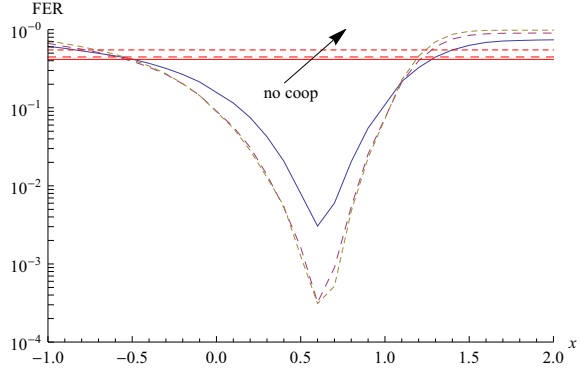

(c) FER along the line containing $\mathrm{S}$ and $\mathrm{D}$ for $L=1,5,130$ (continuous, dotted, dashed).

Fig. 3. FER with uniform power allocation for various relay's positions and $E_{\mathrm{b}} / N_{0}=5 \mathrm{~dB}$. Red lines refer to the case without cooperation.



(a) FER contours for $L=1$.

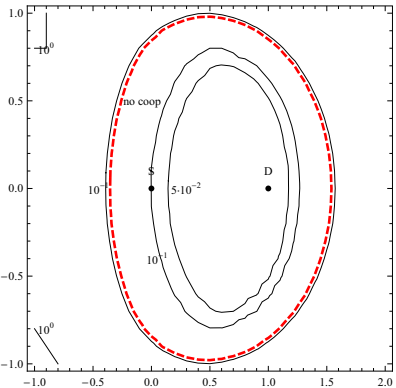

(b) FER contours for $L=5$.

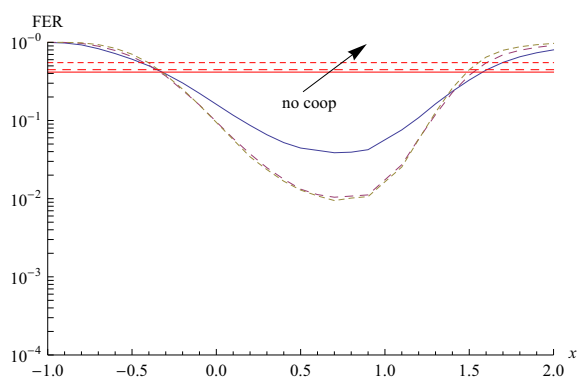

(c) FER along the line containing $\mathrm{S}$ and $\mathrm{D}$ for $L=1,5,130$ (continuous, dotted, dashed).

Fig. 4. FER with ideal power control for various relay's positions and $E_{\mathrm{b}} / N_{0}=5 \mathrm{~dB}$. Red lines refer to the case without cooperation.

to the destination.

\section{CONClusions}

In this paper we analyze relayed communications with cooperative overlay pragmatic space-time codes in block fading channels by considering a real placement of the relay and the quality for the links source-to-destination, source-to-relay, and relay-to-destination when uniform power allocation or ideal power control are assumed. This framework enabled considerations on relay allocation criteria based on a balancing between the frame error rate at the destination and the power level transmitted by the relay depending on its position.

\section{ACKNOWLEDGMENTS}

Research supported by the FP7 European project OPTIMIX (Grant 214625)

\section{REFERENCES}

[1] A. Sendonaris, E. Erkip, and B. Aazhang, "User cooperation diversity. part i. system description," IEEE Trans. Commun., vol. 51, no. 11, pp. 1927-1938, Nov. 2003.

[2] _ - "User cooperation diversity. part ii. implementation aspects and performance analysis," IEEE Trans. Commun., vol. 51, no. 11, pp. 19391948, Nov. 2003.

[3] J. Laneman and G. Wornell, "Distributed space-time-coded protocols for exploiting cooperative diversity in wireless networks," IEEE Trans. Inform. Theory, vol. 49, no. 10, pp. 2415-2425, Oct. 2003

[4] Z. Lin, E. Erkip, and A. Stefanov, "Cooperative regions and partner choice in coded cooperative systems," IEEE Trans. Commun., vol. 54 no. 7, pp. 1323-1334, July 2006.
[5] J. Luo, R. Blum, L. Cimini, L. Greenstein, and A. Haimovich, "Decodeand-forward cooperative diversity with power allocation in wireless networks," IEEE Trans. on Wireless Commun., vol. 6, no. 3, pp. 793 799, March 2007.

[6] A. Stefanov and E. Erkip, "Cooperative coding for wireless networks," IEEE Trans. Commun., vol. 52, no. 9, pp. 1470-1476, Sept. 2004.

[7] M. Dohler, Y. Li, B. Vucetic, A. H. Aghvami, M. Arndt, and D. Barthel, "Performance analysis of distributed space-time block-encoded sensor networks," IEEE Trans. on Veh. Technol., vol. 55, no. 6, pp. 1776-1789, Nov. 2006

[8] J. Laneman, D. Tse, and G. Wornell, "Cooperative diversity in wireless networks: Efficient protocols and outage behavior," IEEE Trans. Inform. Theory, vol. 50, no. 12, pp. 3062-3080, Dec. 2004.

[9] A. Bletsas, H. Shin, and M. Z. Win, "Cooperative communications with outage-optimal opportunistic relaying," IEEE Trans. Wireless Commun., vol. 6, no. 9, pp. 3450-3460, Sep. 2007.

[10] V. Tarokh, N. Seshadri, and A. R. Calderbank, "Space-time codes for high data rate wireless communication: performance criterion and code construction," IEEE Trans. Inform. Theory, vol. 44, no. 2, pp. 744-765, Mar. 1998.

[11] M. Chiani, A. Conti, and V. Tralli, "A pragmatic approach to space-time coding," in Proc. IEEE Int. Conf. on Commun., vol. 9, Helsinki, FI, Jun. 2001, pp. $2794-2799$.

[12] A. Conti, V. Tralli, and M. Chiani, "Pragmatic space-time codes for cooperative relaying in block fading channels," EURASIP J. on Adv. in Sig. Proc., S.I. Wireless Cooperative Networks, vol. 2008, Article ID 872151, 2008.

[13] R.J.McEliece and W.E.Stark, "Channels with block interference," IEEE Trans. Inform. Theory, vol. 30, no. 1, pp. 44-53, Jan. 1984.

[14] A. Stefanov and E. Erkip, "Cooperative space-time coding for wireless networks," IEEE Trans. Commun., vol. 53, no. 11, pp. 1804-1809, Nov. 2005. 